\title{
Les collections techniques, source pour l'historien
}

Essai de méthodologie à travers les collections ferroviaires du XIX ${ }^{\mathrm{e}}$ siècle du Musée des arts et métiers

Technology Collections as Primary Sources: Essay on Methodology from Railway Collections of the Musée des arts et métiers

\section{Lionel Dufaux}

\section{(2) OpenEdition Journals}

\section{Édition électronique}

URL : http://journals.openedition.org/artefact/703

DOI : $10.4000 /$ artefact.703

ISSN : 2606-9245

\section{Éditeur :}

Association Artefact. Techniques histoire et sciences humaines, Presses universitaires du Midi

\section{Édition imprimée}

Date de publication : 15 juin 2017

Pagination : 163-175

ISBN : 978-2-7535-6525-8

ISSN : 2273-0753

\section{Référence électronique}

Lionel Dufaux, «Les collections techniques, source pour l'historien », Artefact [En ligne], 5 | 2016, mis en ligne le 15 novembre 2017, consulté le 09 mars 2020. URL : http://journals.openedition.org/ artefact/703 ; DOI : https://doi.org/10.4000/artefact.703 


\section{Les collections techniques, source pour I'historien Essai de méthodologie à travers les
collections ferroviaires du xix siècle du
Musée des arts et métiers}

Lionel DUFAUX ${ }^{*}$

\section{Résumé}

Les collections ferroviaires du Musée des arts et métiers forment un ensemble patrimonial exceptionnel éclairant l'histoire des techniques mais également le positionnement du Conservatoire des arts et métiers comme vecteur de diffusion des connaissances techniques et de l'innovation quant aux chemins de fer. Véritables sources pour l'historien, ces collections nécessitent néanmoins un traitement particulier pour être interprétables et exploitables : l'élaboration d'outils spécifiques se révèle indispensable et conduisent à une réflexion sur la méthodologie à mettre en œuvre.

Mots-clés : chemins de fer, collections techniques, Conservatoire des arts et métiers, méthodologie, musées, patrimoine.

\section{Abstract. Technology Collections as Primary Sources: Essay on Methodology from Railway Collections of the Musée des arts et métiers}

The railway collections of the Musée des Arts et Métiers are an exceptional heritage enlightening the history of technology and the role of the Conservatoire des Arts et métiers

\footnotetext{
*. Docteur en histoire de l'EHESS, Lionel Dufaux est ingénieur d'études, responsable des collections relatives à l'énergie et aux transports du Musée des arts et métiers. Ses travaux portent sur l'histoire des chemins de fer, des musées et des collections techniques. Il a dirigé le collectif Le Musée des arts et métiers. Guide des collections, Artlys, 2013 et est l'auteur de L'Amphithéâtre, la galerie et le rail, Presses universitaires de Rennes, à paraître en 2017.
} 
for diffusing technical knowledge and innovation regarding the railways. True sources for the historian, these collections require special treatment in order to be interpretable and exploitable: the elaboration of specific tools is indispensable and lead of thinking on the methodology to be implemented.

Keywords : Conservatoire des arts et métiers, heritage, methodology, museums, railways, technical collections.

Avec environ 800 objets, 1400 dessins et 200 photographies, les collections ferroviaires du Musée des arts et métiers forment un ensemble de grande qualité, l'une des premières collections institutionnelles au monde sur le sujet. Les premiers objets et dessins relatifs à des chemins "de bois » ou de fer sont recensés dès les débuts de l'institution, en 1794, tandis que les premières acquisitions sont réalisées en 1824 : la Compagnie du chemin de fer de SaintÉtienne à la Loire s'engageait alors à offrir au Conservatoire des spécimens de moulages de rails importés d'Angleterre en compensation d'une exonération des droits de douane1. À partir de 1832, l'établissement procède à des acquisitions régulières : il effectue des achats de modèles réduits mais également de dessins, qui viennent enrichir son " Portefeuille industriel », et profite des expositions des produits de l'industrie puis des expositions universelles pour actualiser et développer les collections, bénéficiant à ces occasions de crédits spéciaux. Il mobilise enfin un réseau de sociabilités dynamique avec les milieux savants et industriels pour se faire offrir objets et documents pertinents pour ses collections, en particulier après $1850^{2}$.

La position institutionnelle de cette collection, propriété de l'État, affectée au Conservatoire national des arts et métiers, est essentielle. Aujourd'hui constitué sous la forme d'un établissement public dont le statut est proche de celui des universités, le Conservatoire fait partie des créations de la Révolution

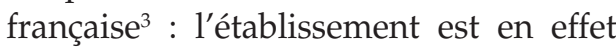
instauré par un décret de la Convention nationale le 19 vendémiaire an III (10 octobre 1794) sur la proposition de l'abbé Grégoire, député du Tiers État. Il s'agit alors de rassembler les outils, machines, modèles, livres, dessins et descriptions utiles au perfectionnement de l'industrie nationale. Le principe du dépôt de machines n'est pas nouveau : Jacques Vaucanson avait en effet réuni à Paris, à l'hôtel de Mortagne, dès les années 1750, une importante collection de machines, outils et livres relatifs à ses travaux quant au perfectionnement de la production des étoffes de soie. Cet ensemble constitue le premier noyau des collections du Conservatoire, auquel vont s'ajouter une partie du cabinet des machines de l'Académie des sciences, au Palais du Louvre, ou les biens d'émigrés placés sous séquestre, conservés notamment à l'hôtel de Montmorency. Le Conservatoire est rapidement doté d'un bureau des dessinateurs (1796), d'un atelier d'entretien des collections (1796), d'une bibliothèque (1798). Les collections rejoignent, en 1798, l'ancien prieuré royal de Saint-Martin-des-Champs, rue 
Saint-Martin à Paris. Les galeries du Conservatoire ouvrent leurs portes en 1802 et l'établissement répond alors à plusieurs missions : il assure la transmission et la diffusion des connaissances techniques et de solutions innovantes par le biais de démonstrations. Il s'agit de mettre en marche des machines ou bien de manipuler des outils, d'en expliquer le fonctionnement, les conditions de construction et d'utilisation, dans une tradition issue de l'Ancien Régime avec une transmission orale et l'imitation du geste (d'où le terme de conservatoire), à l'image du maître et de l'élève ou de l'apprenti. L'idée est de favoriser l'émulation entre artisans et entrepreneurs, de stimuler l'innovation ${ }^{4}$. Le Conservatoire est également chargé d'apporter une expertise aux pouvoirs publics ou aux entreprises en conduisant des expériences ou des essais sur des nouveautés techniques, comme la machine à vapeur, de nouveaux procédés pour la production du papier, ou encore la culture de la betterave sucrière. Ce modèle initial ne semble pas avoir apporté entière satisfaction et le Conservatoire connaît, en 1817-1819, une importante réorganisation : doté d'un conseil de perfectionnement qui supervise son administration et débat des orientations que doit suivre l'institution, il est pourvu, dès 1819, de chaires d'un " haut enseignement de sciences appliquées à l'industrie ", projet en partie esquissé par Charles Dupin, premier professeur de mécanique appliquée ${ }^{5}$. Ces chaires sont confiées à des personnalités du monde savant ou industriel offrant un enseignement ancré dans la pratique, qui apporte ce qu'il faut d'éclairage théorique pour que des hommes de l'art, ouvriers, artisans, contremaîtres, chefs d'ateliers, entrepreneurs, puissent prendre du recul sur leur pratique et parvenir à perfectionner leur production. Tout au long du XIX $x^{\mathrm{e}}$ siècle, le Conservatoire va développer de nouvelles chaires et enrichir ses collections en suivant les progrès de la révolution industrielle dans des secteurs aussi variés que l'agriculture, le textile, la métallurgie, la mécanique, les machines, l'électricité ou encore les chemins de fer ${ }^{6}$ (figure 31, cahier couleur). Il semble qu'une partie de cette collection ait pris une valeur « historique " dès la fin du $\mathrm{XIX}^{\mathrm{e}}$ siècle et, tout au long du $\mathrm{xx}^{\mathrm{e}}$ siècle, cette notion s'est affirmée et a permis de dessiner les contours d'un véritable "musée », au sens actuel du terme, à l'intérieur du Conservatoire. La création du service de muséologie technique après la Seconde Guerre mondiale, la mise en place d'un service pédagogique en 1960, l'organisation des collections en "Musée national des techniques » par Maurice Daumas (19101984) ont en effet contribué à affirmer la valeur muséale et patrimoniale de cette collection. L'important chantier de rénovation, conduit entre 1992 et 2000 dans le cadre des Grands Travaux de l'État, sous la direction de Dominique Ferriot, a non seulement permis de restaurer les espaces d'exposition et d'organiser le parcours de visite en sept domaines thématiques, mais surtout d'établir à La Plaine Saint-Denis de nouvelles réserves offrant l'occasion de redécouvrir des collections, désormais accessibles.

Après avoir étudié plusieurs fonds photographiques relatifs à la traction, au matériel et aux infrastructures ferroviaires ${ }^{7}$, nous avons entrepris un premier recensement des modèles réduits 
de locomotives, voitures et wagons. Une partie de ces collections a été valorisée, en 2007, au Cnam ChampagneArdenne ${ }^{8}$, puis, en 2009, au Musée des arts et métiers ${ }^{9}$, dans le cadre de deux expositions temporaires consacrées à l'histoire de la grande vitesse ferroviaire à l'occasion de l'ouverture de la première section de la ligne à grande vitesse esteuropéenne (figure 32, cahier couleur). Ces expositions ont suscité plusieurs questions liées, notamment, au dépouillement du dernier catalogue imprimé des collections, paru en $1952^{10}$. L'absence de certaines représentations de pièces ou d'éléments significatifs de l'histoire des techniques ferroviaires, la focalisation, à l'inverse, sur certains aspects très pointus et la juxtaposition d'objets et documents de natures variées nous ont conduits à nous interroger sur l'histoire et sur l'utilisation de ces collections ferroviaires ${ }^{11}$. Cherchant à prendre à « rebrousse-poil » ces collections, en mettant au second plan les aspects naturellement traités du point de vue "ferroviphile", comme les compagnies ferroviaires ou les vitesses pratiquées ${ }^{12}$, nous avons formalisé une piste de recherche qui a été le fil conducteur $\mathrm{d}^{\prime}$ 'une thèse de doctorat $\mathrm{d}^{\prime}$ histoire ${ }^{13}$.

Nous savons que le chemin de fer a joué un rôle des plus essentiels dans le développement industriel de la France au XIX ${ }^{\mathrm{e}}$ siècle. Le Conservatoire des arts et métiers, de par ses missions, était désigné comme l'institution gouvernementale la plus à même de soutenir le progrès technique et industriel. Le Musée des arts et métiers conserve aujourd'hui encore une importante collection d'objets et documents relatifs aux chemins de fer. Cela étant posé, on peut s'interroger sur la place tenue par le Conservatoire des arts et métiers, notamment grâce à ses collections, dans la diffusion des connaissances techniques et des innovations concernant le secteur ferroviaire tout au long du XIX siècle. En d'autres termes, quelle a été l'utilité du Conservatoire des arts et métiers face à la " révolution ferroviaire ", pour reprendre l'expression de Georges Ribeill1 ${ }^{14}$. À travers ce questionnement, ce n'est pas tant une histoire des chemins de fer qui nous intéressait en premier lieu, mais bien une histoire du Conservatoire des arts et métiers et de son positionnement face au monde savant et industriel étudiée par le prisme du développement des chemins de fer en France.

L'étude des collections ferroviaires était la première étape pour apporter des éléments de réponse à ce questionnement. Mais la nature particulière de ces sources-objets et sources-images allait nous amener à conduire une réflexion particulière sur le traitement de ce matériel pour tenter de construire une méthodologie adaptée ${ }^{15}$. Cet " essai de méthodologie » vise, à partir de l'expérience conduite avec les collections ferroviaires du Musée des arts et métiers, à formaliser une proposition d'étude, de traitement et d'analyse des collections techniques considérées comme sources pour l'historien. 


\section{Constituer le corpus}

Il a fallu, dans un premier temps, établir la liste des objets et documents concernés, en procédant au dépouillement systématique des sources « de gestion » et en effectuant l'inventaire descriptif des collections.

\section{Les sources de gestion}

On désigne par cette expression l'ensemble des documents qui nous renseignent sur la gestion des collections du musée. Ces sources doivent être confrontées et décryptées pour nous délivrer des informations quant à l'évolution des pratiques muséales au sein de l'institution ${ }^{16}$. Entrent dans cette catégorie les registres d'inventaire, désignés par l'expression « mains courantes »: il s'agit de registres sur lesquels sont consignés, pour chaque objet, le numéro d'inventaire, la désignation, et, dans certains cas, la modalité d'acquisition et la date d'entrée en collection. Deux mains courantes se succèdent : la première couvre la période 1849-1884 ; la seconde 1884-1972. Ces sources essentielles permettent, en théorie, de retracer l'histoire des acquisitions (et des éventuelles sorties), et d'identifier la composition des collections. Toutefois, la première main courante a été établie de manière rétrospective : les acquisitions antérieures à 1849 y sont consignées sans forcément respecter l'ordre d'entrée ou sans préciser les modalités d'acquisitions. De même, les registres n'ont pas toujours été tenus avec la plus grande rigueur, d'où des informations contradictoires ou erronées. Il a donc fallu mettre en perspective ces registres avec d'autres sources, comme les catalogues imprimés des collections, qui offrent des " photographies » de l'état de la collection à un moment donné, deux inventaires intermédiaires, établis en $1837^{17}$ et $1842^{18}$, ou encore des archives comptables pour la période antérieure à 1850, comprenant des factures, des inventaires partiels ou des listes d'objets radiés des collections et versés à l'Administration des domaines ${ }^{19}$.

\section{Les sources de gouvernance}

Il s'agit des documents, plus habituels, relatifs à la gestion de l'établissement. Entrent ici les procès-verbaux des séances du Conseil de perfectionnement $^{20}$, qui concernent, pour certaines d'entre elles, les collections : ou encore, les courriers de remerciements pour des dons qui peuvent confirmer les modalités d'acquisition, prévoir les démarches engagées par l'institution pour développer les collections, ou comprendre l'entrée ou non de tel ou tel objet.

\section{L'inventaire descriptif}

Nous avons, à peu près dans le même temps, procédé à l'inventaire descriptif des objets, à rapprocher des opérations légales de récolement des collections qui s'imposent à tous les musées labellisés « Musée de France » ${ }^{21}$. Il s'agit, pour chaque objet ou document, de s'assurer de l'existence du bien, de son statut juridique, de son état sanitaire. L'inventaire descriptif est une étape fondamentale qui permet de prendre conscience de la matérialité des collections : chaque objet est mesuré, ses matériaux consti- 
tutifs identifiés, son état de conservation évalué, ses inscriptions relevées. On s'assure, à ce moment-là, que les objets ou documents repérés lors du dépouillement des sources de gestion entrent bien dans le corpus thématique. L'inventaire descriptif doit être réalisé avec le plus grand soin, car il permet de collecter des informations importantes : quel est l'objet/document? A-t-on affaire à un modèle ou à un objet de grandeur réelle? Quels sont les matériaux constitutifs? Quel soin apporté à la construction? Quelles sont les inscriptions? Quel est l'état? On se rapproche ici de la « critique externe » formalisée à la fin du xIX ${ }^{e}$ siècle par Charles-Victor Langlois et Charles Seignobos, et visant à décrire les documents ${ }^{22}$.

\section{Étudier le corpus}

Il importe, une fois les objets et documents repérés et identifiés, d'apporter des éléments d'analyse afin de comprendre pourquoi ils sont entrés, à un moment donné, dans les collections et quelle était leur place par rapport à l'histoire générale des chemins de fer.

\section{Les typologies}

Cette étude passe, dans un premier temps, par la qualification des typologies présentes. La collection comporte des objets en trois dimensions et des documents. Mais on peut y distinguer plusieurs sous-catégories : ainsi, on notera les objets de " grandeur réelle ", c'est-à-dire de provenance industrielle, et les modèles, éventuellement à échelle réduite et pouvant présenter des aménagements particuliers comme des coupes ou des mises en peinture pour souligner tel ou tel organe. La question des dimensions est essentielle : la taille d'une locomotive à vapeur (et son coût) n'ont pas permis au Conservatoire de présenter de telles machines; en revanche, les modèles réduits, moins encombrants, ont offert une solution pertinente permettant en outre d'insister par des coupes sur certains organes; a contrario, les manipulateurs de télégraphie sont présents dans les collections en taille réelle. Il faut également distinguer les représentations " complètes » des éléments constitutifs : à côté des modèles de locomotives, représentations complètes, on trouve des "pièces détachées ", également sous forme de modèles réduit ou de grandeur naturelle, selon les cas, qui viennent éclairer des points particuliers (figure 33, cahier couleur). Cette dichotomie complet/partiel se retrouve dans les documents, qu'il s'agisse de dessins, de tableaux ou de photographies.

\section{Les provenances}

Il importe également de déterminer les modalités d'acquisition : les objets et documents ont-ils été acquis à titre onéreux, sur les deniers de l'établissement, ou bien ont-ils été offerts? Les sources de gouvernance relatives aux budgets permettent de considérer l'importance des expositions universelles qui offrent 
autant d'opportunités d'acquisitions en raison des crédits supplémentaires octroyés à l'établissement ${ }^{23}$. Pour les collections ferroviaires, on constate de nombreux dons à partir de 1850, signe du développement de réseaux de sociabilités que les professeurs entretiennent avec le monde savant et le monde industriel; on peut ainsi solliciter des dons et se faire offrir, à moindres frais, objets et documents utiles à l'institution.

\section{Le contexte technique}

Bien que familiarisé avec l'histoire des chemins de fer, nous avons procédé à un dépouillement assez important de sources imprimées afin de situer les objets et documents de la collection dans l'histoire générale des techniques ferroviaires. Nous avons ainsi traité près de 800 références : il s'agit principalement d'articles du Bulletin de la Société d'encouragement pour l'industrie nationale, des Mémoires et comptes rendus de la Société des ingénieurs civils, de la Publication industrielle de Jacques Eugène Armengaud, $\mathrm{du}$ Portefeuille économique des machines de Charles Alfred Oppermann, de la Revue générale des chemins de fer; mais également des publications des expositions universelles; et, surtout, des très nombreux traités et ouvrages techniques ferroviaires, parmi lesquels figurent le Traité élémentaire des chemins de fer de Perdonnet, le Portefeuille de l'ingénieur des chemins de fer de Perdonnet et Polonceau, du Guide $d u$ constructeur conducteur de locomotive de Le Châtelier, entre autres. Grâce aux informations recueillies dans ces sources et à leur confrontation avec les collections, nous avons pu déterminer quelle avait été la place des collections ferroviaires du Conservatoire vis-à-vis de l'innovation. Une approche analytique et critique devenait dès lors possible, permettant de comprendre, pour chaque objet, son importance par rapport à l'histoire des chemins de fer. Quelle est sa place dans l'innovation ferroviaire? Marque-t-il une rupture? Quelles étaient ses qualités? Pourquoi a-t-il été retenu dans les collections? Pour quoi faire? Comment est-il entré dans les collections, par quel biais et à quel moment? On est ici dans une opération de "critique interne » et de "critique de provenance», pour laquelle il faut mobiliser les sources de gestion et de gouvernance, la bibliographie et les sources imprimées.

\section{Le catalogue raisonné}

Les données collectées sont, par la nature des sources consultées, extrêmement hétérogènes. Pour les classer et les exploiter le plus finement possible, nous avons élaboré un catalogue raisonné, outil habituel de l'étude des collections mais finalement peu utilisé pour les collections techniques. Ce catalogue nous a permis d'appréhender chaque objet ou document selon plusieurs points de vue : typologie (à quel type de représentation avons-nous affaire?); identification (de quoi s'agit-il, qui est le «concepteur » de l'invention représentée et l'auteur - constructeur, dessinateur, photographe... - de sa représentation?); acquisition (quand l'objet ou le document est-il entré dans les collections, par quels biais?); usages (à quoi l'objet ou le document pouvait-il servir dans les collections?); utilité (quelle est la place de l'objet ou du document dans l'histoire des techniques ferroviaires?). 
Les trois cent cinquante notices de ce catalogue ont permis d'initier un traitement quantitatif et qualitatif des collections : nous avons pu déterminer le volume d'objets et documents relatifs aux chemins de fer, mettre en évidence des phases d'acquisitions, déterminer des ensembles et sous-ensembles thé- matiques (traction, matériel roulant, infrastructures et installations fixes, équipements des ateliers ferroviaires, exploitation), mais également voir l'importance de ces collections par rapport à l'univers ferroviaire, dans quelle mesure elles ont été mobilisées par les enseignements du Conservatoire.

\section{Au-delà des collections : vision globale et questionnement historien}

Une fois les collections étudiées et classées dans le catalogue raisonné, nous avons été confronté à une limite sérieuse : chaque objet ou document était analysé, pièce à pièce, mais il restait difficile d'avoir une vision globale de la collection, et, au-delà, de l'établissement. Compte tenu de la nature même $\mathrm{du}$ Conservatoire et de son histoire, il semblait donc pertinent de confronter les données recueillies avec d'autres corpus.

\section{Traitement statistique et provenances}

Nous avons, dans un premier temps, opéré plusieurs traitements quantitatifs, essayant de déterminer si, pour les locomotives, les voitures et wagons ou les infrastructures, le ratio entre objets de grandeur réelle et modèles ou celui entre objets et dessins était pertinent. Nous avons également cherché à voir s'il y avait des données transversales intéressantes, par exemple en effectuant des recensions par compagnie ferroviaire, constructeur, provenance géographique. Force est de constater que les résultats obtenus à partir de ces grilles n'étaient pas concluants.

Le traitement thématique $a$, en revanche, permis de faire émerger une catégorie importante qui ne paraissait pas évidente, relative à l'organisation $\mathrm{du}$ travail, aux outils, machines-outils et fonctionnement des ateliers ferroviaires.

Autre point intéressant, l'étude des provenances a confirmé l'importance des expositions universelles et la part des dons après 1850. Les bulletins et mémoires de la Société d'encouragement et de la Société des ingénieurs civils ont révélé les « filtres » apposés par les professeurs du Conservatoire qui appartenaient à ces réseaux, et ont permis de constater la démarche suivie pour actualiser les leçons et les collections.

\section{Les enseignements}

L'étude des enseignements techniques du Conservatoire a été une clef essentielle pour mettre les collections en perspective à l'intérieur même de l'établissement. Le dépouillement $\mathrm{d}$ 'archives où figurent les programmes des leçons a été extrême- 
ment important. Il a d'abord révélé l'absence d'une chaire dédiée aux chemins de fer : l'opportunité en a été longuement débattue à la fin du XIX ${ }^{\mathrm{e}}$ siècle au sein du Conseil de perfectionnement, mais cette chaire n'a jamais vu le jour. Son absence, à mettre en regard de l'existence d'une collection ferroviaire, interroge. Ce sont en fait les cours de mécanique appliquée qui ont largement traité les questions ferroviaires. Par chance, nous disposons de plusieurs cours imprimés : ceux de Charles Dupin, au début des années $1820^{24}$; ceux d'Arthur Morin, au début des années $1840^{25}$; ceux, enfin, d'Henri Tresca, à la fin des années $1850^{26}$. Il est ainsi possible de voir l'organisation $\mathrm{du}$ cours et la place tenue par les chemins de fer, tant de point de vue des infrastructures que de la traction. On découvre ainsi la manière dont le chemin de fer était perçu au Conservatoire. La position particulière du Conservatoire comme établissement d'enseignement technique a pu être précisée. Celle-ci se distingue des écoles d'ingénieurs, des écoles techniques ou encore des dispositifs de formation propres aux compagnies ferroviaires. Il ne s'agit pas, pour le Conservatoire, d'interférer avec ces différents établissements, mais bien de proposer, à un public composé d'ouvriers qualifiés, de contremaîtres, de chefs d'ateliers ou d'entrepreneurs, des connaissances leur permettant de perfectionner leur pratique et d'évoluer dans leur carrière professionnelle. Ces connaissances s'appuient sur des exemples concrets puisés dans des résultats d'expériences ou d'après la pratique sur le terrain. Elles ne visent pas l'exhaustivité ou un niveau très pointu, mais se veulent plus généralistes. Le Conservatoire n'avait en effet pas vocation à former aux métiers spécifiquement ferroviaires, mais à transmettre des connaissances utiles pour que ses auditeurs et visiteurs puissent progresser, entre autres, dans leur carrière au sein de l'industrie ferroviaire. Les questions relatives aux techniques ferroviaires ont été principalement traitées dans les leçons de mécanique appliquée, et c'est sans doute pour cette raison qu'un cours de chemin de fer n'a pas vu le jour. La collection d'objets, de tableaux et de photographies vient compléter cet enseignement, soit en illustrant directement le propos des leçons, soit pour les visiteurs qui viennent les étudier par eux-mêmes dans les galeries. Le Conservatoire participe à la formation de mécaniciens, de chaudronniers, d'ajusteurs qui reçoivent des éléments pratiques quant à la construction de chaudières, de mécanismes divers, d'outils qui peuvent entrer dans le cadre ferroviaire sans lui être spécifiques. Le chemin de fer, vu comme un ensemble d'applications de la mécanique est l'une des caractéristiques du Conservatoire. On peut ainsi expliquer le nombre limité, dans les collections constituées au xIx ${ }^{e}$ siècle, de représentations de locomotives ou de wagons complets : l'établissement ne cherche pas à les recenser, mais simplement à illustrer, à travers quelques exemples significatifs, les grands principes qui président à leur conception, à leur construction et à leur entretien.

\section{Les ressources}

Nous avons également procédé au dépouillement des fonds de la bibliothèque centrale. Service établi très peu de temps après la création du Conservatoire, 
la bibliothèque conserve une collection d'ouvrages et de périodiques; les registres $d$ 'inventaire, à rapprocher des sources de gestion du musée, révèlent que les fonds ont été, pour l'essentiel, constitués par achats, en suivant de très peu la publication. On peut en déduire que le Conservatoire était attentif à l'innovation dans le domaine ferroviaire, et qu'il cherchait à constituer un fonds de référence dans le domaine.

De la même manière, nous avons dû considérer le Portefeuille industriel à part : bien qu'appartenant aux collections et ayant le même statut que les objets, les dessins du Portefeuille étaient en effet gérés séparément au XIX ${ }^{e}$ siècle. Il s'agissait alors d'une «bibliothèque de dessins » plutôt destinée aux ingénieurs et à un personnel qualifié assurant un encadrement intermédiaire (chefs d'ateliers, contremaîtres, chefs de bureaux d'études), que l'on pouvait venir consulter sur place, dans une salle de lecture. Ils n'étaient pas destinés à être exposés dans les galeries ou à illustrer les leçons des professeurs.

\section{Le Conservatoire et I'innovation}

Souvent qualifié de manière simpliste et rapide de musée "de l'innovation » ou " de prototypes », le Conservatoire des arts et métiers considère l'innovation de manière nette, ce qui le conduit à n'évaluer et à ne diffuser que certaines d'entre elles. La mise à jour des collections, entre autres lors des expositions universelles, et la convocation, dans les leçons, d'exemples faisant écho à des expériences ou à des essais récents traduisent la place du Conservatoire sur le front de l'innovation. Les très nombreuses représentations d'éléments constitutifs de locomotives ou d'infrastructures font état des progrès récents, le plus souvent sanctionnés avec succès par l'expérience. Il ne s'agit pas de faire entrer dans les galeries ou de montrer dans les amphithéâtres ce qui, sur le papier, paraît séduisant, mais bien ce qui répond aux attentes des constructeurs et des exploitants ferroviaires. Le Conservatoire apporte son expertise aux pouvoirs publics et aux entreprises, notamment par l'intermédiaire de ses laboratoires. Il exerce cette mission en s'appuyant sur un réseau de sociabilités dynamique qui lui permet de se tenir au courant des dernières innovations dans le secteur, d'avoir des échos et des retours d'expérience, d'apposer un « filtre » pour déterminer les éléments à relayer dans les leçons ou dans les collections. Ce réseau repose sur l'implication des professeurs et du directeur dans les jurys des expositions industrielles, dans les sociétés savantes ou sur des échanges réciproques avec le monde industriel, dont les compagnies ferroviaires et les constructeurs mécaniques. Avec un corps professoral constitué de personnalités du monde savant, le Conservatoire est un pivot, qui combine transmission de connaissances techniques et illustrations de lois et principes théoriques.

L'étude des enseignements, des collections et des ressources révèle par ailleurs un fonctionnement cohérent où ces différents éléments se complètent et se répondent. Étudiés jusqu'à présent séparément, enseignements et collections, mis en résonance à travers le prisme de la thématique ferroviaire, retrouvent leur articulation logique. Les collections d'objets, de tableaux et de photogra- 
phies apparaissent alors comme des supports de l'enseignement technique : elles viennent expliquer le fonctionnement d'un procédé, illustrer son application ou ses utilisations.

\section{Conclusions}

\section{Affiner l'approche des collections}

Ce travail nous a permis d'appréhender les collections du Musée des arts et métiers avec plus de subtilité, en abandonnant l'angle d'attaque « ferroviphile ", qui butait sur certaines limites. En replaçant les collections dans leurs contextes technique et muséographique, nous sommes parvenus à comprendre comment les objets et documents avaient été acquis. Nous avons pu distinguer les fonds en fonction de leurs usages, qu'il s'agisse des collections présentées dans les galeries, des fonds de la Bibliothèque ou encore du Portefeuille industriel. Nous avons également pu comprendre l'articulation des objets entre eux : représentations complètes et éléments constitutifs se répondent et ne visent pas à former un fonds exhaustif, traitant l'ensemble de l'évolution des techniques ferroviaires, mais insistant sur certains procédés que l'on retrouve dans ce secteur à différentes époques, et sur les innovations marquantes qui font évoluer le système selon les attentes et les besoins des industriels. Les leçons viennent, de la même manière, exposer et expliquer ces principes à des auditeurs inscrits dans la pratique, qui viennent, grâce à des éléments théoriques illustrés, perfectionner leur activité profes- sionnelle. La dimension transversale de certaines connaissances techniques transmises soulignent des convergences entre filières techniques et insistent sur la place de la mécanique appliquée.

Cette étude a également été l'occasion d'identifier plusieurs " couches » qui, superposées les unes aux autres, gênent la compréhension et la lisibilité des collections. L'édition de 1952 du catalogue des collections relatif aux transports sur rails mettait ainsi quasiment sur un pied d'égalité les objets acquis au début du XIX ${ }^{\mathrm{e}}$ siècle et ceux entrés plus récemment dans le cadre d'une collaboration avec la SNCF insistant sur l'histoire et sur le futur des chemins de fer, le tout étant classé selon une perception chronologique et thématique qui n'existait pas à l'origine des collections (figure 34, cahier couleur). Il a donc fallu « déconstruire » cet édifice pour saisir la philosophie qui a présidé à la constitution des collections ferroviaires, en particulier au $\mathrm{XIX}^{\mathrm{e}}$ siècle, et sortir - temporairement - du discours didactique de l'exposition permanente du musée.

\section{Méthodologie}

En s'appuyant sur une approche appliquée à un cas précis, ce travail nous a permis de réfléchir aux moyens à mettre en œuvre pour l'étude et le traite- 
ment des collections, considérées comme sources historiques, et de voir comment il convenait de les confronter à d'autres corpus. Le catalogue raisonné semble ainsi être une solution pratique pour documenter, critiquer et classer les collections, mais il paraît important de rappeler la nécessité de saisir les contextes institutionnel et technique pour que les collections ne soient pas seulement des illustrations, mais bien des sources à part entière. Pour ce qui concerne le Conservatoire, cela conduit donc à s'interroger sur les enseignements, sur les instances de direction de l'institution, sur les ressources proposées ou encore sur les implantations géographiques.

\section{Pistes futures}

La rénovation du musée, opérée dans les années 1990, a conduit à la présentation d'une quinzaine de pièces ferroviaires dans le dernier des "domaines" thématiques, consacré aux transports. Érudits et amateurs de la chose ferroviaire ne peuvent que regretter la disparition de la salle des chemins de fer qui, bien que devenue obsolète depuis la fin des années 1970, avait le mérite de faire honneur à cette page d'histoire des techniques. Pour autant, la mise en perspective des chemins de fer avec d'autres modes de transports était une autre manière de renouer - en partie - avec ce qui avait été le grand principe suivi depuis les années 1830 quant à la perception des chemins de fer au Conservatoire : longtemps considérés comme une application de la mécanique parmi d'autres, les chemins de fer étaient de nouveau intégrés dans une approche plus globale. Le travail d'inventaire et l'élaboration d'un catalogue raisonné nous ont permis de mettre en évidence la qualité de la collection dans son ensemble, et l'importance des éléments constitutifs, tout aussi parlants que les modèles complets du point de vue de l'innovation. En ce sens, il est aujourd'hui possible de construire une politique scientifique et culturelle autour de la question ferroviaire qui permette de valoriser ce pan oublié des collections auprès d'un plus large public, tout en procédant à de nouvelles acquisitions qui actualiseraient les collections tout en respectant leur nature intrinsèque. 


\section{Notes}

1. Archives historiques du Musée des arts et métiers, S77.

2. Marie-Sophie Corcy, « Exposer l'invention », Musée des arts et métiers, la revue, $\mathrm{n}^{\circ}$ 51-52, 2010, p. 78-87.

3. Bruno Belhoste, « La Révolution et l'éducation ", Musée des arts et métiers, la revue, $\mathrm{n}^{\circ} 49,2008$, p. 20-31; Dominique Poulot., Une histoire des musées de France. $\mathrm{XVIII}^{e}-\mathrm{XX}$ siècles, Paris, La Découverte, 2005 ; id., « Le modèle républicain du musée et son héritage ", Musée des arts et métiers, la revue, $\mathrm{n}^{\circ} 49$, 2008, p. 8-19.

4. On parle de « conservatoire » comme lieu de transmission de techniques (les « arts ») et de savoir-faire (les «métiers ») par observation et imitation du geste.

5. Carole Christen et François Vatin (dir.), Charles Dupin (1784-1873). Ingénieur, savant, économiste, pédagogue et parlementaire du Premier au Second Empire, Rennes, PUR (Carnot), 2009, p. 11-28.

6. Lionel Dufaux (dir.), Le Musée des arts et métiers. Guide des collections, Paris, Musée des arts et métiers/Artlys, 2013; Alain MERcier, Un Conservatoire pour les arts et métiers, Paris, Gallimard (Découvertes), 1993.

7. Lionel Dufaux, « La gare Saint-Lazare : une histoire en images ", Musée des arts et métiers, la revue, $\mathrm{n}^{\circ} 45,2006$, p. 47-54; id., "Voyage ferroviaire en banlieue ouest ", Musée des arts et métiers, la revue, $\mathrm{n}^{\circ} 48,2008$, p. 55-62.

8. Gérard Villermain-Lécolier (dir.), Petite Vitesse deviendra grande, Reims, Cnam Champagne-Ardennes (Les cahiers des soirées scientifiques), 2007.

9. Toujours plus vite! Les défis du rail, Paris, Musée des arts et métiers / La Vie du Rail, 2009.

10. Conservatoire national des arts et métiers. Catalogue du musée. Section DB. Transports sur rails, Paris, Conservatoire national des arts et métiers, 1952.

11. Lionel Dufaux, «Approche historique des collections ferroviaires du Conservatoire des arts et métiers, support de l'enseignement technique ", Documents pour l'histoire des techniques, $\mathrm{n}^{\circ} 20,2^{\mathrm{e}}$ semestre 2011, p. 147-166; id., « Panorama des collections ferroviaires du Musée des arts et métiers », Historail, n 32, janvier 2015, p. 76-87.

12. Lionel Dufaux, « La vitesse, critère discutable pour la constitution de collections ferroviaires. L'exemple des collections du Musée des arts et métiers ", Revue d'histoire des chemins de fer, $\mathrm{n}^{\circ}$ 43-44, 2012, p. 93-108.
13. Lionel Dufaux, L'Amphithéâtre, la galerie et le rail. Le Conservatoire des arts et métiers, ses collections et le chemin de fer : diffusion des connaissances techniques et de l'innovation au XIX $\mathrm{X}^{e}$ siècle, thèse de doctorat, sous la dir. de Liliane Hilaire-Pérez, EHESS, Paris, 2015.

14. Georges Ribeill, La Révolution ferroviaire, Paris, Belin, 1993.

15. Liliane Hilaire-PÉrez, "Invention technique et corpus de sources », in Marie-Sophie Corcy, Christiane Demeulenaere-Douyère et Liliane Hilaire-PÉrez (dir.), Les archives de l'invention. Écrits, objets et images de l'activité inventive, Toulouse, Méridiennes, 2003, p. 9-25.

16. Marie-Sophie Corcy, « La politique d'enrichissement des galeries du Conservatoire des arts et métiers, 1849-1880 ", Artefact, 2015, n³, p. 165182.

17. « Catalogue des Modèles exposés dans les Galeries du Conservatoire qui sont présentement ouvertes au Public », [5 mai] 1837, Archives nationales [ensuite AN], F/12/4866.

18. «Copie de l'Inventaire Général des objets existant au Conservatoire Royal des Arts \& Métiers, fait par la Commission nommée à cet effet Par Mr Le Ministre du Commerce ", 1842, AN, F/12/4866. Nous remercions très vivement Christiane Demeulenaere-Douyère et MarieSophie Corcy pour la communication et l'étude de ces documents.

19. «État des objets réformés au Conservatoire des arts et métiers à livrer au Domaine au profit de l'État », 1850, Arch. du Musée des arts et métiers, $10^{\circ} 567$.

20. Arch. du Cnam, 2AA.

21. Loi $\mathrm{n}^{\circ}$ 2005-5 du 5 janvier 2002 relative aux Musées de France, et Code du patrimoine.

22. Charles-Victor LANGLOIs et Charles SeIgnobos, Introduction aux études historiques, Paris, Hachette, 1898.

23. Marie-Sophie Corcy, « La politique d'enrichissement des galeries du Conservatoire des arts et métiers (1849-1880) », art. cit.

24. Charles DupIN, Géométrie et méchanique des arts et métiers et des beaux-arts. Cours normal à l'usage des Artistes et des Ouvriers, des Sous-Chefs et des Chefs d'ateliers et de manufactures; Professé au Conservatoire royal des arts et métiers, Paris, Bachelier, 1826-1828, 3 vol.

25. Arthur Morin, Leçons de mécanique pratique à l'usage des auditeurs des cours du Conservatoire des arts et métiers, et des sous-officiers et ouvriers d'artillerie, Paris, Mathias, 1846-1853, 4 vol.

26. Arthur Morin et Henri Tresca, Mécanique pratique. Des machines à vapeur. Production de la vapeur, Paris, Hachette, 1863. 